\title{
$R R N 6$ and $R R N 7$ encode subunits of a multiprotein complex essential for the initiation of rDNA transcription by RNA polymerase I in Saccharomyces cerevisiae
}

\author{
Daniel A. Keys, Loan Vu, Joan S. Steffan, Jonathan A. Dodd, Robert T. Yamamoto, Yasuhisa Nogi, ${ }^{1}$ \\ and Masayasu Nomura
}

Department of Biological Chemistry, University of California, Irvine, California 92717-1700 USA; ${ }^{1}$ Department of Biochemistry, Saitama Medical School, Moroyama, Iruma-gun, Saitama 350-04, Japan

\begin{abstract}
Previously, we have isolated mutants of Saccharomyces cerevisiae primarily defective in the transcription of 35S rRNA genes by RNA polymerase I and have identified a number of genes ( $R R N$ genes) involved in this process. We have now cloned the $R R N 6$ and $R R N 7$ genes, determined their nucleotide sequences, and found that they encode proteins of calculated molecular weights of 102,000 and 60,300 , respectively. Extracts prepared from rrn6 and rrn7 mutants were defective in in vitro transcription of rDNA templates. We used extracts from strains containing epitope-tagged wild-type Rrn6 or Rrn7 proteins to purify protein components that could complement these mutant extracts. By use of immunoaffinity purification combined with biochemical fractionation, we obtained a highly purified preparation (Rrn6/7 complex), which consisted of Rrn6p, Rrn7p, and another protein with an apparent molecular weight of 66,000 , but which did not contain the TATA-binding protein (TBP). This complex complemented both rrn6 and rrn 7 mutant extracts. Template commitment experiments carried out with this purified Rrn6/7 complex and with rrn6 mutant extracts have demonstrated that the Rrn6/7 complex does not bind stably to the rDNA template by itself, but its binding is dependent on the initial binding of some other factor(s) and that the Rrn6/7 complex is required for the formation of a transcription-competent preinitiation complex. These observations are discussed in comparison to in vitro rDNA transcription systems from higher eukaryotes.
\end{abstract}

[Key Words: rDNA transcription; transcription factor; RNA polymerase I; yeast; RRN6 gene; RRN7 gene]

Received July 7, 1994; revised version accepted August 11, 1994.

In eukaryotic cells, large rRNA is synthesized by transcription of tandemly repeated rRNA genes by RNA polymerase I ( $\mathrm{Pol}$ I) in the nucleolus. The mechanism of this transcription process has been studied mainly by use of a variety of in vitro transcription systems, from organisms such as Acanthamoeba, Xenopus, mouse, rat, and human (for review, see Sollner-Webb and Mougey 1991; Reeder 1992; Paule 1994; Moss and Stefanovsky 1994). These studies have identified cis-acting DNA elements, as well as trans-acting protein factors, which together with Pol I participate in the initiation of rRNA gene transcription. The general picture that has emerged from these studies is that the gene promoter, which covers $\sim 150$ bp upstream from the transcription initiation site and extends slightly into the transcribed region, consists of two domains, a core domain around the transcription initiation site and an upstream control element (UCE), and that at least two transcription factors, UBF (upstream binding factor), and SLl, are required for accurate initiation at the rDNA gene promoter. UBF interacts with both the UCE and the core domain and helps the binding of SL1 [promoter selectivity factor (Bell et al. 1988); also called by other names; e.g., TIF-IB (Schnapp and Grummt 1991; Radebaugh et al. 1994)]. The genes for UBF have been cloned from several vertebrates, including humans (Jantzen et al. 1990), rats (O'Mahony and Rothblum 1991), mice (Hisatake et al. 1991), and frogs (Bachvarov and Moss 1991; McStay et al. 1991) and their sequence analyses have demonstrated the presence of domains related to the chromosomally associated proteins HMG1 and HMG2. SL1 was purified first from human (Comai et al. 1992) and then from mouse (Eberhard et al. 1993) and Acanthamoeba (Radebaugh et al. 1994), and the presence of TBP (TATA-binding protein) has been demonstrated. In addition to TBP, SLl complexes from human (Comai et al. 1992) and mouse (Eberhard et al. 1993) were shown to contain three polypeptide subunits [TBP-associated factors (TAFs)], but characteriza- 
tion of these TAFs, including cloning and sequencing of their genes, has not yet been reported. Although both UBF and SL1 are required for stable initiation complex formation in some systems (McStay et al 1991), in other systems SLl alone can recognize the gene promoter /and make a stable complex) and UBF is only stimulatory (Smith et al. 1990; Schnapp and Grummt 1991). Thus, in analogy to well-studied RNA polymerase II (Pol II) and III (Pol III) transcription systems, SL1 appears to be the essential transcription factor corresponding to TFIID (for Pol II) and TFIIIB (for Pol III) that is responsible for correctly positioning Pol I at the start site; UBF may be an assembly factor analogous to TFIIIC and TFIIIA (for the Pol III tRNA and 5S RNA gene systems) or to some DNA-binding proteins (for some Pol II systems that do not utilize the TATA element; see, e.g., Pugh and Tjian 1992; Hernandez 1993). Under certain in vitro assay conditions, rDNA transcription appears to take place in the complete absence of UBF (Smith et al. 1993).

In addition to UBF and SL1, other Pol I transcription factors have been reported. For example, by use of a mouse in vitro system, Grummt and co-workers showed that two additional factors, TIF-IA and TIF-IC, are required for specific initiation at the mouse rDNA promoter (Schnapp and Grummt 1991). This conclusion is in contrast to some other systems, for example, the human system or the Acanthamoeba system, where purified UBF, SL1, and Pol I are apparently sufficient for accurate initiation (Comai et al. 1992; for review, see Paule 1994). Of course, differences in experimental systems and methods of analyses might be responsible for such a discrepancy. It is also possible that the various factor preparations or Pol I preparations used in these studies were not sufficiently purified and biochemically defined, leading to apparent discrepancies in the results obtained in different laboratories. It should also be noted that requirements for these transcription factors defined by in vitro experiments have not been clearly demonstrated for rDNA transcription in vivo except perhaps for TBP (Cormack and Struhl 1992).

Previously, we have initiated genetic approaches to dissect and study components involved in rDNA transcription by Pol I by use of the yeast Saccharomyces cerevisiae. Specifically, we devised a system to screen for mutants that are preferentially defective in rRNA synthesis by Pol I (Nogi et al. 1991a). This screening system utilizes the discovery (Nogi et al. 1991b) that functional rRNA can be produced from a fusion gene (pGAL7-35S rDNA, which consists of the 35S rRNA coding region fused to the GAL7 promoter) by Pol II. Mutants (rrn mutants) that could not grow on glucose media but could grow on galactose media because of transcription of the fusion gene (on a plasmid) by Pol II were isolated and classified into 11 complementation groups (Nogi et al. 1991a; Nomura et al. 1993); and 1 new complementation group (L. Vu, K. Sutton, R. Yamamoto, and M. Nomura, unpubl.). Analysis of the RNA synthesized after pulselabeling of cells with $\left[{ }^{3} \mathrm{H}\right]$ uridine has demonstrated that rRNA synthesis by Pol I is defective in these mutants, but Pol II and Pol III functions are relatively unaffected.
Three of the $R R N$ genes identified in this way $(R R N 1$, $R R N 2$, and $R R N 4$ ) were shown to encode Pol I-specific subunits, A190, A135, and A12.2, respectively (Nogi et al. 1991a, 1993), confirming the validity of the genetic strategies and the results of in vivo uridine incorporation experiments. [It should be noted that the $S$. cerevisiae Pol I has been well characterized and the genes for all the 14 subunits, except for A14, have now been cloned and sequenced/Thuriaux and Sentenac 1992; Nogi et al. 1993).] The six of the remaining eight $R R N$ genes $(R R N 3$, RRN5, RRN6, RRN7, RRN9, and RRN10) have been cloned and sequenced and do not appear to code for Pol I subunits (this paper; L. Vu, Y. Nogi, R. Yamamoto, E. Fantino, L. Burson, T. Nguyen, and M. Nomura, unpubl.). One approach to study the function of proteins encoded by these genes is to study extracts prepared from mutants regarding their rDNA transcription activity. Although faithful in vitro transcription of rDNA by Pol I with extracts from the yeast $S$. cerevisiae was developed only recently (Riggs and Nomura 1990; Lue and Kornberg 1990; Schultz et al. 1991), and biochemical fractionation of the extracts has not been advanced compared to the other systems mentioned above, extracts from some of these mutants have proved to be useful in defining components involved in rDNA transcription by Pol I. In this paper, we first describe cloning and sequencing of the RRN6 and RRN7 genes, and then describe in vitro experiments demonstrating that the encoded proteins, Rrn6p and Rrn7p, represent components of a multisubunit transcription factor essential for the initiation of rDNA transcription by Pol I.

\section{Results \\ Cloning and characterization of the RRN6 and RRN7 genes}

Among the many rrn mutants independently isolated so far, six mutants belong to a complementation group that defines the gene $R R N 6$, and two mutants belong to another that defines the gene RRN7 (Nogi et al. 1991a; L. $\mathrm{Vu}, \mathrm{S}$. Gehrung, Y. Nogi, and M. Nomura, unpubl.). A mutant strain carrying rrn6-1 (isolation number 195) and another strain carrying rrn7-1 (isolation number 801) were used for cloning of the RRN6 and RRN7 genes. These mutant strains carry plasmid pNOY103, require the expression of the plasmid-encoded pGAL7-35S rDNA fusion gene for growth, and cannot grow on glucose media as a result of repression of the fusion gene. Because of the tighter phenotype of these mutants at $36^{\circ} \mathrm{C}$ relative to $30^{\circ} \mathrm{C}, 36^{\circ} \mathrm{C}$ was used for gene cloning experiments. A yeast genomic DNA bank based on the YCpNI vector (CEN3 ARS1 TRP1) was used to transform these $r r n 6$ and $r r n 7$ mutants (which are trp1), and $\operatorname{trp}{ }^{+}$ transformants that could grow on glucose plates were isolated. Plasmids were recovered from these candidate transformants and examined further. In this way, we isolated genomic DNA clones containing RRN6, which complements the rrn6-1 mutation, and clones containing $R R N 7$, which complements the rmn 7-1 mutation. 
Physical structures of the chromosomal DNA regions containing RRN6 and RRN7 are shown in Figure 1, and amino acid sequences of encoded proteins Rrn6p and Rrn7p, as deduced from the DNA sequences, are given in Figure 2. Formal proof that the cloned genes correspond to RRN6 and RRN7 defined by the original genetic experiments was obtained by identifying amino acid alterations in some of the original mutants (data not shown).

$R R N 6$ encodes a protein of 894 amino acids with a calculated molecular weight of 102,000 and a calculated isoelectric point of 4.9. As expected from the phenotype of the rrn6 mutants, standard gene disruption experiments demonstrated that RRN6 is an essential gene. A diploid strain (a derivative of NOY397) carrying the disrupted rrn6 gene (rrn6::HIS3; see Fig. 1b), together with one intact gene (RRN6) on the other chromosome, pro-

a

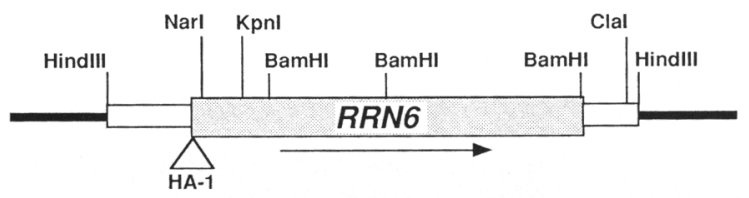

b

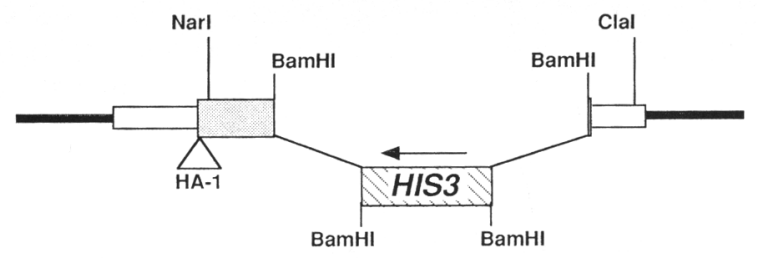

C

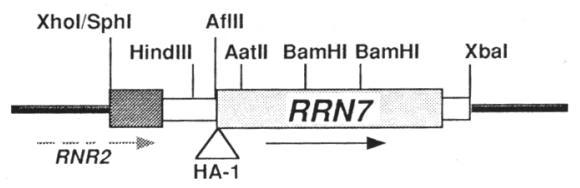

d

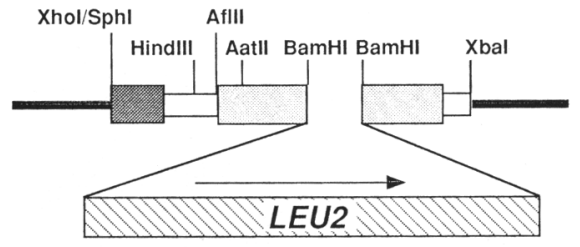

Figure 1. Restriction enzyme maps of the RRN6 and the RRN7 regions. (a) The HindIII fragment carrying RRN6 in pNOY204; (b) the DNA fragment in pNOY3193 that was used to construct the rrn6::HIS3 null allele; $(c)$ the SphI-XbaI fragment carrying $R R N 7$ in pNOY265; $(d)$ the DNA fragment in pNOY3166 that was used to construct the rrn 7::LEU2 null allele. Protein coding regions of $R R N 6$ and $R R N 7$ are stippled as is the distal part of $R N R 2$ upstream of RRN7. Positions of the HAl epitope-encoding oligonucleotide inserted in some plasmids for epitope tagging (see Table 1) are indicated by open triangles. Restriction enzyme sites relevant to plasmid and strain constructions are shown. Arrows show the direction of transcription. duced, upon sporulation, tetrads with two viable (his3) and two nonviable (presumed to be HIS3) spores. After we completed these experiments, a paper appeared reporting the DNA sequence of a restriction fragment from a region on the left arm of $S$. cerevisiae chromosome II (Skala et al. 1992). We found that the DNA sequence of $R R N 6$ is identical to the open reading frame YBL03.12 described in this paper. However, these investigators described a gene disruption experiment and stated that YBL03.12 is not essential for growth. It is not clear whether this discrepancy is attributable to the difference in the method of gene disruption or the difference in the strain background.

RRN7 encodes a protein of 514 amino acids with a calculated molecular weight of 60,300 and a calculated isoelectric point of 8.1. Standard gene disruption experiments confirmed that the gene is essential for growth. From the DNA sequence upstream of $R R N 7$, we found that $R R N 7$ is located downstream of a known gene, $R N R 2$, which encodes the small subunit of ribonucleotide reductase and is centromere linked on chromosome X (Elledge and Davis 1987); these two genes are transcribed in the same direction and their protein coding regions are separated by 369 nucleotides (Fig. 1c).

Examination of amino acid sequences of Rrn6p and Rrn7p has not revealed any unique features of these proteins. There is a leucine zipper-like motif near the amino terminus of Rrn6p (underlined in Fig. 2a), but similarity to the bZIP motif found in quite a few DNA-binding proteins (Vinson et al. 1989) is not strong and its significance is unclear. There is also a short segment in Rrn7p where several glutamine $(Q \mid$ residues are clustered [shown in bold in Fig. 2b], but this glutamine richness is not as striking as that found in several known glutamine-rich transcription factors (see, e.g., Courey and Tjian 1988).

We have constructed haploid strains with the chromosomal RRN6 (or RRN7) disrupted and carrying the pGAL7-35S rDNA fusion on a plasmid. These strains (NOY567 and NOY558) can grow on galactose, but not on glucose, and fail to synthesize rRNA by Pol I in vivo (data not shown). These strains were utilized to make the mutant extracts used for the in vitro experiments to be described below. We have also constructed haploid strains with chromosomal RRN6 (or RRN7) disrupted and carrying a HAl epitope-tagged $R R N 6$ (or $R R N 7$ ) gene on a centromere plasmid (NOY552 and NOY573; see Materials and methods). We found that the presence of the HAl epitope at the amino terminus of Rrn6p for Rrn7p) does not apparently interfere with the normal function of Rrn6p (or Rrn7p) and that these strains grow at the same growth rate as control RRN6 (or RRN7) strains. These strains were utilized to detect and affinity purify Rrn6p (or Rrn7p) with monoclonal antibodies against the HAl epitope.

Complementation of $\mathrm{rrn} 6$ and $\mathrm{rrn} 7$ mutant extracts in vitro

Figure 3a summarizes fractionation of yeast extracts 
a

\section{Rrn6p}

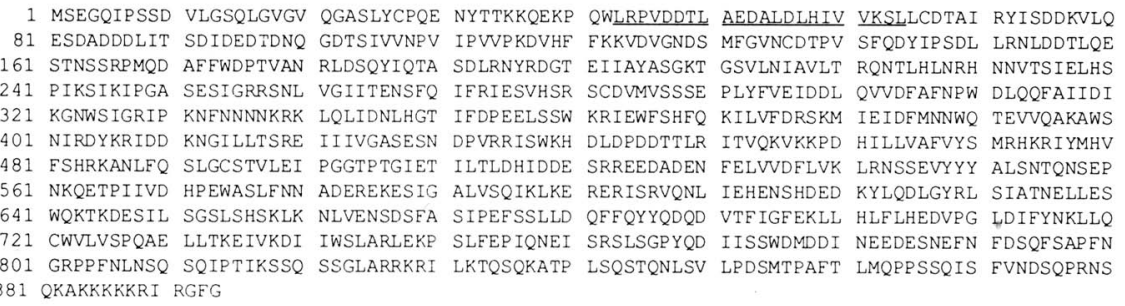

Figure 2. Predicted amino acid sequences of Rrn6p and Rrn7p. The leucine zipperlike motif present in Rrn6p is underlined, and the glutamine (Q)-rich region (71-87) in $\operatorname{Rrn} 7 \mathrm{p}$ is shown in bold. The nucleotide sequences of RRN6 and RRN7 have been submitted to the GenBank data base under accession numbers L33863 and L33864, respectively.

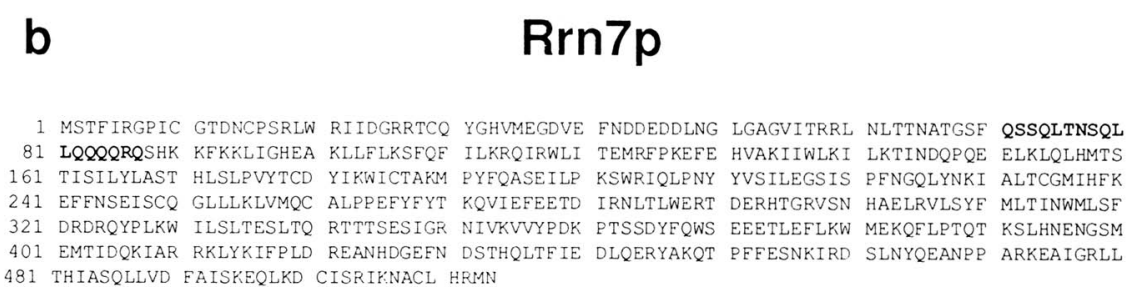

b Rrn7p used for in vitro transcription reactions. Extracts were prepared from wild-type and mutant strains as described in Materials and methods. For extracts prepared from wild-type yeast cells, efficient transcription of rRNA in this system requires the combination of two fractions, a PC-300 fraction (eluting from the phosphocellulose column at $300 \mathrm{~mm} \mathrm{KCl}$ ) and a D-300 fraction (eluting from the DEAE column at $300 \mathrm{~mm} \mathrm{KCl}$; Fig. 3b, lane 3). The majority of Pol I in the extracts is present in the PC-300 fraction and little in the D-300 fraction, as determined by Western blot analysis with antibodies against the largest subunit of Pol I. However, the PC-300 fraction could not be replaced by our purified Pol I preparation, suggesting the presence of a transcription factor(s) in addition to Pol I in this fraction (data not shown). Extracts prepared from either rrn6 or $r m 7$ mutant strains are inactive in specific Pol I transcription in vitro (Fig. 3b, lanes 4,7$)$. This activity can be complemented by the addition of the D-300 fraction from wild-type cells (Fig. $3 \mathrm{~b}$, lanes 5,8$)$. These results indicate that the mutant extracts are missing or are defective in an essential Pol I transcription factor (or factors) that is normally present in the D-300 fraction. Complementation of activity in the mutant extracts serves as a biochemical assay for this missing factor(s). RRN6 activity therefore refers to the fraction or factor(s) from wild-type extracts that will complement activity in the rrn6 mutant extract in vitro. Similarly, RRN7 activity refers to the fraction or factor(s) that will complement activity in the $r m 7$ mutant extract.

RRN6 activity was further fractionated by heparinagarose chromatography followed by gel filtration as shown in Figure 3a. The activity was found in the H-400 fraction, that was then analyzed by Superose 6 gel-filtration chromatography. RRN6 activity was found to elute as a single peak of activity, with an estimated native molecular weight of $\sim 450,000$ [Fig. $3 c_{\text {; }}$ the molecular weight was estimated from the elution pattern of reference proteins (thyroglobulin, $\beta$-amylase, and bovine serum albumin) under the identical condition (data not shown|. The estimate by glycerol gradient centrifugation, however, gave a smaller value (see below)]. Because the Rrn6p is predicted to have a molecular weight of 102,000 , these results indicate that the Rrn6p is either multimeric, highly modified post-translationally, or part of a multiprotein complex.

The same fractions described above were also assayed for RRN7 activity; RRN7 activity was found to cofractionate with RRN6 activity, eluting from the heparinagarose column at $400 \mathrm{~mm} \mathrm{KCl}$, and eluting from the Superose 6 column as a single peak of activity with an estimated native molecular weight of $\sim 450,000$ (Fig. 3c; see the comments above). These results suggest that Rrn6p and Rrn7p may be components of a single multiprotein complex. To confirm the presence of both Rrn6p and $\mathrm{Rrn} 7 \mathrm{p}$ in the fractions that complement both $r \mathrm{rn} 6$ and $r m 7$ mutant extracts, fractionation experiments were repeated with yeast strains expressing HAl epitope-tagged Rrn6p or Rrn7p (HA1-Rrn6p or HA1Rrn7p|. An H-400 fraction was prepared from a strain expressing HAl-Rrn6p and subjected to Superose 6 gel filtration as described above. Column fractions were then pooled, concentrated, resolved by SDS-PAGE, and analyzed by Western blot with an anti-HAl antibody. In this way, (epitope-tagged) Rrn6p was detected in the column fractions corresponding to the peak of RRN6/7 activity (data not shown). Likewise, an extract prepared from a strain expressing HAl-Rrn7p was similarly fractionated, and Superose 6 fractions were analyzed by Western blot. Rrn7p was also detected in the fractions corresponding to the peak of RRN6/7 activity /data not shown). The association of Rrn6p and Rrn $7 p$ in a single complex has proven to be correct by experiments to be described in later sections. 


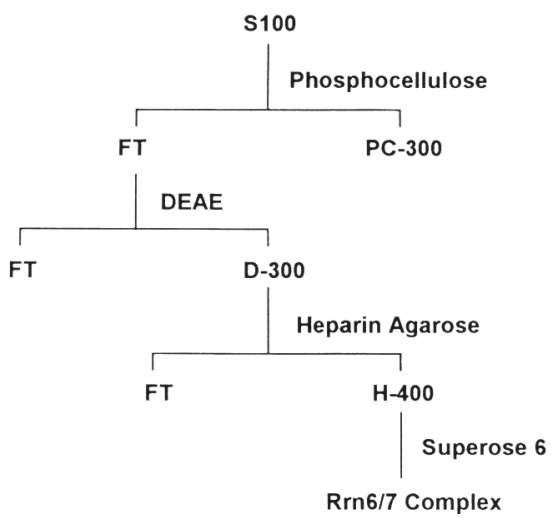

b

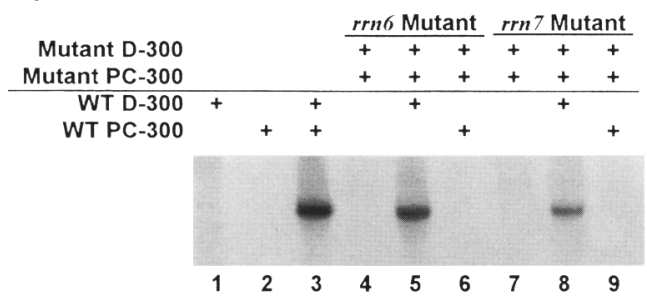

C

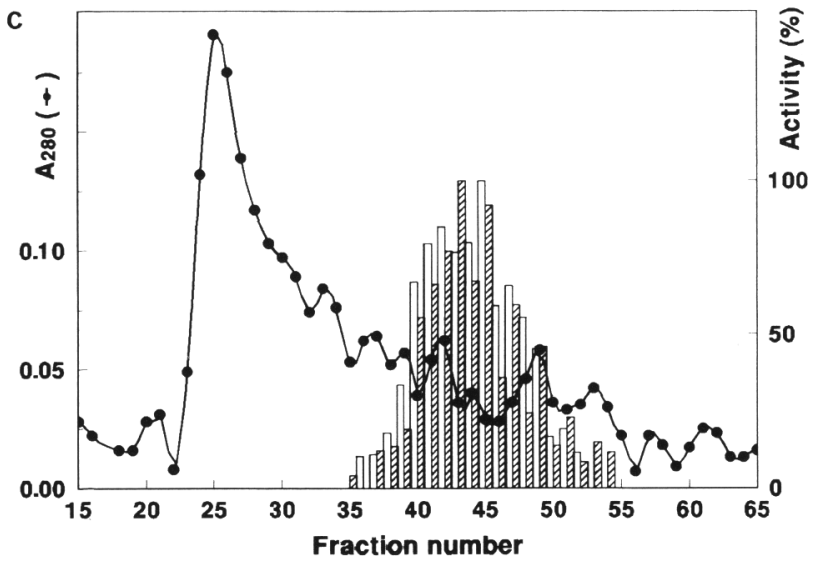

Figure 3. Complementation of $r r n 6$ and $r r n 7$ mutant extracts by wild-type protein fractions in vitro. $(a)$ Fractionation of yeast extracts used for in vitro transcription reactions. $|b|$ Transcriptional activity of wild-type and mutant extracts. Extracts prepared from wild-type (JHRY20-2C $\Delta 1$ ) or from mutant strains (NOY567 and NOY558) of yeast were assayed for specific Pol I transcription in vitro. The reactions contained $\sim 20 \mu \mathrm{g}$ each of the indicated D-300 fraction and/or $\sim 2 \mu \mathrm{g}$ each of the indicated PC-300 fraction. (c) Superose 6 column chromatography for fractionation of $R R N 6$ and $R R N 7$ activities. A wild-type $\mathrm{H}-400$ fraction (from NOY573 expressing HA1-Rm6p in this case) was loaded onto the column. Aliquots from individual fractions were assayed for RRN6 and for RRN7 activities. The results were quantitated by autoradiograph densitometry and plotted as open bars for RRN6 activity and hatched bars for RRN7 activity. The values are normalized to the highest activity observed among fractions. (O) Protein concentrations measured by absorbance at $280 \mathrm{~nm}$.

Because of a likely possibility that the complex containing both Rrn6p and Rrn7p (called Rrn6/7 complex hereafter without defining other associated protein components) might correspond to the transcription factor SLl studied in vertebrate systems, we asked whether TBP is associated with this complex. Superose 6 fractions described above were analyzed by Western blot with a polyclonal antibody against yeast TBP. TBP could be detected in the fractions corresponding to the peak of RRN6/7 activity. However, TBP was also detected in the fractions analyzed that followed these Rrn6/7 peak fractions, and no clear answer was obtained to this question (data not shown; see below and Discussion).

\section{Affinity purification of the Rrn6/7 complex}

As further evidence that Rrn6p and Rrn7p are part of a single complex, coimmunoprecipitation experiments were performed with epitope-tagged Rrn6p or Rrn7p to determine whether precipitation or affinity purification of Rrn6p could specifically precipitate RRN7 activity, and vice versa. D-300 fractions containing HAl-tagged Rrn6p were treated with an affinity resin consisting of HA1-specific monoclonal antibody chemically crosslinked to protein A-Sepharose. After washing, epitopetagged complexes were specifically eluted with competing HAl peptide. This eluate was then assayed in an in vitro transcription reaction for RRN6 and RRN7 activity. The eluate was found to be able to complement both rrn6 and rrn7 mutant extracts, indicating that RRN7 activity can be coimmunoprecipitated with the epitopetagged Rrn6p (data not shown; see below). Similarly, D-300 fractions containing HA1-tagged Rrn7p were subjected to immunoprecipitation with the same antibodycross-linked protein A-Sepharose, and eluates were found to complement both $r r n 6$ and $r r n 7$ mutant extracts (data not shown; see below), indicating that Rrn6p can be coimmunoprecipitated with epitope-tagged Rrn $7 p$. Control preparations obtained from untagged wild-type extracts did not show such complementation activity.

The above affinity purification procedure was adopted to purify the Rrn6/7 complex on a larger scale. Extracts were prepared in parallel from three strains: a strain expressing HAl-Rrn6p (NOY573), a strain expressing HAl-Rrn7p (NOY552), and a control strain without any HAl epitope tagging (NOY418). After the first affinity precipitation-elution step, the Rm6/7 complex was recovered free from $\mathrm{HAl}$ peptide by binding to a heparinagarose column at $300 \mathrm{~mm} \mathrm{KCl}$ followed by elution at $600 \mathrm{~mm} \mathrm{KCl}$. A second affinity purification was then carried out, and the final preparations were examined for protein composition by use of SDS-PAGE (see Fig. 4a,b) and for their activities to complement rrn6 and $r r n 7 \mathrm{mu}-$ tant extracts (Fig. 4c).

As can be seen from the silver-stained protein patterns of the gels shown in Figure 4a, (lanes 2,3), the Rrn6/7 complex appears to consist of three polypeptides of apparent molecular weights of $115,000,66,000$, and 56,000 . The 115-kD band represents Rrn6p (calculated molecular weight of 102,000 ) as indicated by a shift of the band to a slightly higher molecular weight in the preparation containing HAl-Rrn6p and by a positive staining in 
Figure 4. Affinity purification of Rrn6/7 complex. Rrn6/7 complexes were purified from strain NOY573 expressing HA1-Rrn6p (HA1-Rrn6p complex) or strain NOY552 expressing HAI-Rrn7p (HAl-Rrn7p complex) with anti-HAl protein ASepharose, heparin-agarose chromatography, and anti-HAl protein $\mathrm{G}-$ Sepharose, as described in $\mathrm{Ma}$ terials and methods. Control preparation was obtained from the strain (NOY418) without HAl tagging. These three preparations were analyzed by SDSPAGE followed by silver staining of protein $(a)$ and by Western blot analysis with anti-HAl antibody $(b)$. Activities of these preparations to complement rrn6 and $r r n 7$ extracts were also analyzed by in vitro transcription $(c)$. Lanes in $a$ and $b$ are as follows: (Lane 1) Untagged control; (lane 2) HAl-Rrn6p complex; (lane 3) HAl-Rm7p complex. Positions of molecular weight markers are indicated. In $c$, transcription was carried out with D-300 plus PC-300 fractions obtained from rrn6 and $\mathrm{rrn} 7$ mutant extracts supplemented with various preparations as indicated. The absence of complementation by mixing of $\operatorname{rrn} 6$ and $r r n 7$ extracts is also shown in lane 3 . It should be noted that the transcription activity obtained by complementation of $r r n 7$ extracts with the Rrn6/7 complex is always somewhat weaker than that obtained by complementation of rrn6 extracts, as can be seen here (cf. lane 5 with lane 4; see also Fig. 3b, cf. lane 8 with lane 5). When PC-300 from $r r n 7$ mutants was replaced by that from the wild-type, the complementing activity was as high as that observed for the rrn6 system. It appears that $r m 7$ mutations lead to (indirect) inactivation of some factor(s) present in PC-300 that is different from Rrn7p. It should also be noted that the samples used for silver staining $(a)$ were concentrated by precipitation with TCA before the analysis, whereas the samples used for Western blot analysis $(b)$ were without such a concentration step. The reason why the Rrn $7 \mathrm{p}$ band is split into a doublet in silver staining and not in the Western blot analysis is not known but might be related to this acid treatment. Alternately, the resolution into the doublet might have been lost in the step of transfer of proteins from the SDS gel to the immobilon P (PVDF).

Western blot with the anti-HAl antibody. Similarly, the 56-kD band represents $\operatorname{Rrn} 7 \mathrm{p}$ (calculated molecular weight of 60,000$)$. This band often splits into two bands, as can be seen in Figure 4, and one must be a derivative of the other, possibly reflecting a specific protein modification, because both appear to shift to slightly higher molecular weights in the preparation containing HAlRrn7p (see comments in the legend to Fig. 4). We do not know the nature of the difference between these two forms of Rrn7p, nor do we know the reason for differences between the apparent molecular weights $(115,000$ and 56,000$)$ and the calculated molecular weights $(102,000$ and 60,000) observed for Rrn6p and Rrn7p.

The $66-\mathrm{kD}$ band is present in both preparations containing HAl-Rrn6p or HAl-Rrn7p but is completely absent in the control untagged (mock-purified) preparation, and is therefore likely to be a genuine component of the Rrn6/7 complex. We did not see any other bands that are present in the Rrn6/7 complex but absent in the control preparation. The protein of particular interest, TBP $(27$ $\mathrm{kD}$, was not observed. Furthermore, TBP could not be detected by Western blot with polyclonal antibodies against yeast TBP (data not shown). These results indicate that TBP is not a component of the purified Rrn6/7 complex, although it is still possible that the Rrn6/7 complex is associated with TBP in less-purified fractions or in vivo. From relative intensities of silver-stained bands (and antibody-stained Rrn6p and Rrn $7 p$ bands), the Rrn6/7 complex appears to consist of three proteins,
Rrn6p, the 66-kD protein (p66), and Rrn7p, which appear to be present in equal molar ratios.

The Rrn6/7 complex preparations purified in this way complemented both rrn6 (Fig. 4c, lanes 4,8,9) and rrn7 mutant extracts (Fig. 4c, lane 5), whereas the mock-purified control preparation had no complementation activities (Fig. 4c, lane 7). Finally, the highly purified Rrn6/7 complex (carrying HAl-Rrn7p) was subjected to glycerol gradient centrifugation, and fractions were analyzed for activity to complement an rrn6 mutant extract and for HAl-Rrn7p (with anti-HAl antibody) as well as Rrn6p (with polyclonal antibodies against Rrn6p) by Western blot. Both complementation activity and the proteins (Rrn6p and HAl-Rrn7p) cosedimented, giving a peak at the same position, and this peak position corresponded to the size of $\sim 200-\mathrm{kD}$ as judged from protein size markers sedimented in parallel (data not shown). These results support the conclusion further that Rrn6p and $\operatorname{Rrn} 7 \mathrm{p}$ are components of a single (stable) multiprotein complex consisting of Rrn6p, Rrn7p, and p66, which is required for rDNA transcription. In connection with this conclusion, it should be noted that $r m 6$ and $r m 7$ mutant extracts do not complement each other in vitro (Fig. 4c, lane 3), even though rrn6 and rrn7 mutants complement in vivo (Nogi et al. 1991a). It appears that Rrn6p and Rrn7p may exist mostly as Rrn6/7 complex in vivo, and that in the absence of other components of the complex (due to mutation), these proteins cannot be recovered in extracts (PC-300 and D-300 fractions) from the 
mutant strains, or if they exist in extracts, the free proteins fail to reconstitute an active Rrn6/7 complex under the experimental conditions used.

\section{Participation of Rrn6/7 complex in formation of a transcription-competent initiation complex}

Both the previous in vivo experiments (Nogi et al. 1991a) and the present in vitro experiments described above demonstrate that the Rrn6/7 complex is essential for rDNA transcription, but they do not delineate steps in transcription where function of the complex is required. To study this question, we carried out template commitment analysis to find out whether Rrn6/7 participates in the formation of a transcription-competent preinitiation complex.

As demonstrated in other Pol I transcription systems, and as was also the case in our previous studies with unfractionated extracts from wild-type yeast cells (Kulkens et al. 1991), we found that when an $\mathrm{rrn} 6$ or $\mathrm{rrn} 7$ extract supplemented with the purified Rrn6/7 complex is preincubated with a template DNA in the absence of nucleoside triphosphates, some essential transcription factor(s) forms a stable complex with this first template and does not exchange onto a subsequently added second template (Fig. 5a, lanes 3,4). When the purified Rrn6/7 complex was omitted from the preincubation and added immediately following the second template, the first template was still preferentially transcribed to the exclusion of the second, indicating that a stable preinitiation complex is able to form on the first template even in the absence of the Rrn6/7 complex (Fig. 5a, lanes 7,8). Conversely, when the purified Rrn6/7 complex alone was preincubated with the first template and the rrn6 mutant extract was not added until immediately following the addition of the second template, both templates were transcribed with roughly equal efficiency (Fig. 5a, lanes $9,10)$. The same results were also obtained when the experiments were repeated with the first template preincubated with the purified Rrn6/7 complex plus TBP (data not shown). These results demonstrate that a factor (or factors) other than the Rrn6/7 complex is responsible for primary binding to the rDNA template. The overall level of transcription was reduced when the Rrn6/7 complex alone was preincubated with the first template (Fig. $5 a$, lanes 9,10 ; see the legend); this is likely the result of its loss by nonspecific binding to tube walls or to DNA during the $60-\mathrm{min}$ preincubation in the absence of cell extracts.

Although the results of the template commitment assay described above indicate that the Rrn6/7 complex is not the primary DNA-binding protein that recognizes the rRNA promoter, it is still possible that the Rrn6/7 complex forms a stable association with the promoter by binding to a protein-DNA complex that is an intermediate to the formation of a complete preinitiation complex. To examine this possibility, a variation of the template commitment assay was performed as shown in Figure $6(a, b)$. In this assay, two templates were preincubated separately with the mutant rrn6 extract, and limiting amounts of the highly purified Rrn6/7 complex was added to only one of the templates. If the Rrn6/7 complex is able to form a stable association with the template, then it should not exchange onto the second template during the second preincubation when both templates are mixed. This was observed for the Rrn6/7 complex (Fig. 6). Taken together, the results of the template commitment analyses shown in Figures 5 and 6 indicate that the Rrn6/7 complex does not bind stably to the rDNA template by itself, but its binding is dependent a

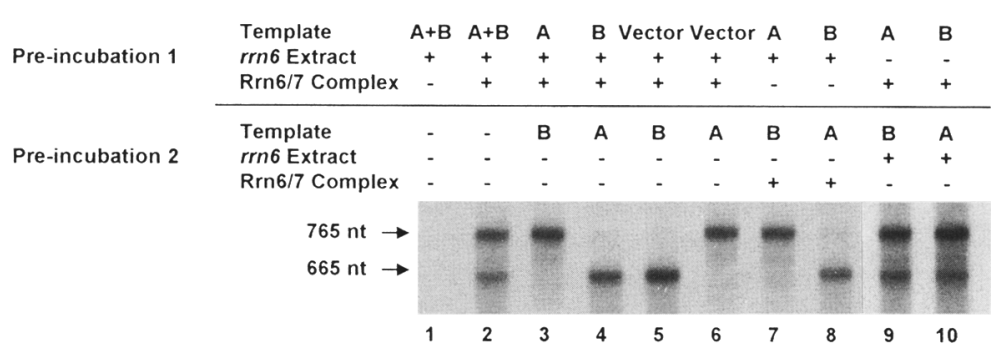

b

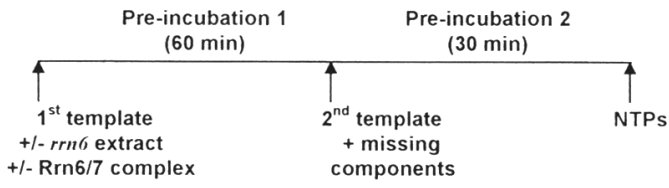

Figure 5. Formation of a committed template-factor complex in the absence of Rrn6/7 complex. Template commitment experiments were carried out with rrn6 mutant extract (D-300 + PC-300 fractions) and Rrn6/7 complex (containing HAl-Rrn6p), as outlined in $b$. The mutant extract and Rrn6/7 complex together or individual fractions were preincubated with the first template for $60 \mathrm{~min}$ followed by the addition of the second template together with fractions that were omitted during the first preincubation. After an additional $30 \mathrm{~min}$ of incubation, nucleoside triphosphates (NTPs) were added, and transcription was carried out for $40 \mathrm{~min}$. RNA was analyzed by urea-PAGE followed by autoradiography $(a)$. (Lanes 9 and 10 are from an autoradiograph with an exposure time that is approximately three times longer than that for the remaining lanes.) Two templates were used: Template A is pSIRT, which is expected to give a 765-nucleotide transcript; template B is pSIRT $\Delta+27 /+128$, which is expected to give a 665-nucleotide transcript. In lanes 5 and 6, the rm6 extract and Rrn6/7 complex were preincubated with nonspecific DNA (400 ng of pBluescript DNA) followed by the addition of rDNA template as indicated. Each assay contained, where indicated, $400 \mathrm{ng}$ (each) of template DNA, $\sim 20 \mu \mathrm{g}$ of D-300 fraction, and $\sim 2 \mu \mathrm{g}$ of PC-300 fraction prepared from the rrn6 mutant and $10 \mu \mathrm{l}$ of an appropriately diluted, affinity-purified Rrn $6 / 7$ complex preparation (containing HAl-Rrn6p; see Fig. 4). 
Keys et al.

a

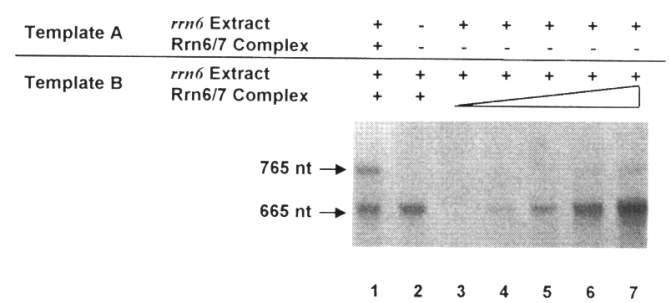

b

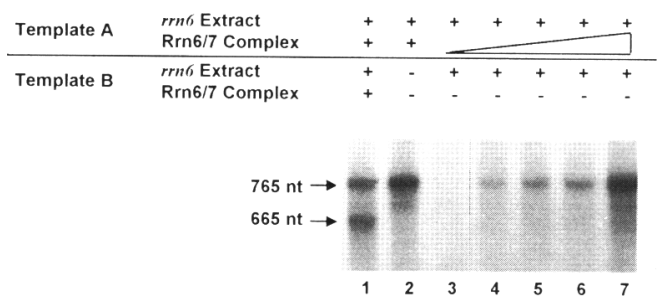

c

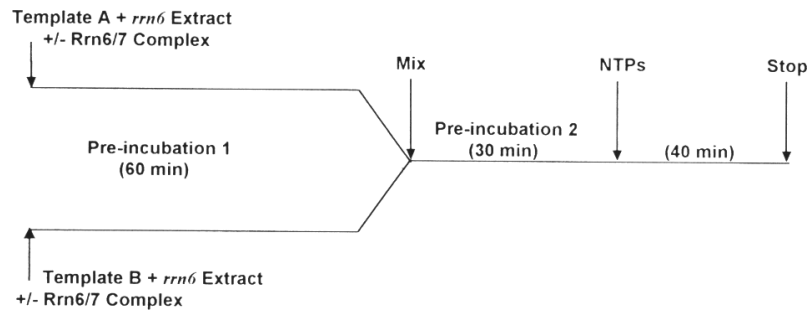

Figure 6. A stable association of Rrn6/7 complex with rDNA template. Two different rDNA templates (template A, pSIRT; template B, pSIRT $\Delta+27 /+128 ; 400 \mathrm{ng}$ each) were incubated separately with buffer (one of the templates in lane 2 in $a$ and $b$ ), with equal amounts of $r m 6$ mutant extract $(\sim 20 \mu \mathrm{g}$ of D-300 + $\sim 2 \mu \mathrm{g}$ of $\mathrm{PC}-300$ ) with or without supplement of increasing amounts of affinity-purified Rrn6/7 complex to one of the templates, as indicated. After a $60-\mathrm{min}$ preincubation, the two extract/template mixtures were combined and incubated for an additional $30 \mathrm{~min}$ (preincubation 2). Transcription was initiated by the addition of nucleoside triphosphates. After a 40-min reaction, RNA products were analyzed by urea-PAGE. Autoradiographs are shown in $a$ and $b$, and an outline of the experiments is shown in $c$. The reaction mixtures contained the amounts of Rrn6/7 complex corresponding to $0,0.005,0.01,0.02$, and 0.04 $\mu \mathrm{l}$ \{lanes 3, 4, 5, 6, and 7, respectively), and $0.04 \mu \mathrm{l}$ (lanes 1 and 2) of an affinity-purified preparation (containing HAl-Rrn6p; see Fig. 4), and total reaction volume was $40 \mu \mathrm{l}$ after mixing.

on the initial binding of some other factor(s), and that the Rrn6/7 complex is required for the formation of a transcription-competent preinitiation complex.

\section{Discussion}

By isolating mutants that are specifically defective in the transcription of rDNA by Pol I, we have so far defined 11 $R R N$ genes. We have now demonstrated that two of these genes, RRN6 and RRN7, encode subunits of a multiple protein transcription factor essential for the initia- tion of rDNA transcription in vitro. We have purified this transcription factor, the Rrn6/7 complex, and found that it contains a protein with an apparent molecular weight of 66,000 (p66) in addition to Rrn6p and Rrn7p. We used a combination of genetic and biochemical approaches. Thus, the initial cloning and sequencing of these genes allowed epitope tagging of the genes, that was extremely useful for immunoaffinity purification of the gene products as well as their detection. Mutant extracts also exhibited a specific defect(s) in in vitro rDNA transcription that could be used for unambiguous demonstration of the activity of the purified Rrn6/7 complex and for defining a step in which this complex participates in transcription initiation.

The size of the purified Rrn6/7 complex estimated by glycerol gradient centrifugation is $\sim 200 \mathrm{kD}$. Although the size of this highly purified complex was not estimated by sizing column chromatography because of the scarcity of the preparation, the size of the complex present in D-300 or $\mathrm{H}-400$ fractions was estimated by both sizing columns and glycerol gradient centrifugation. By use of the same molecular weight standard proteins, the former method gave the size of $\sim 450 \mathrm{kD}$ and the latter method gave $\sim 200 \mathrm{kD}$, the same value as that obtained for the purified complex. Although we do not know the reason for discrepancy in the estimates between the two methods used (an asymmetric shape of the complex being a possibilityl, it appears that the Rrn6/7 complex is not associated with additional components in crude protein fractions in any stable way that might affect its sedimentation behavior in glycerol gradient centrifugation. The sum of the molecular weights of Rrn6p (102,000 from the sequence and 115,000 from SDS-PAGE analysis), p66, and Rrn7p $(60,000$ from the sequence and 56,000 from SDS-PAGE analysis) is $\sim 230,000$, which is somewhat higher than the value for the complex obtained with the centrifugation method, but is not inconsistent with this value in view of the lower estimate of the centrifugation method relative to the sizing column method. From these considerations, we tentatively conclude that the Rrn6/7 transcription factor is a heterotrimer consisting of three proteins, Rrn6p, p66, and Rrn7p. However, it is possible that this heterotrimer further dimerizes and exists as a hexamer under the conditions used for sizing column chromatography.

As described in the introductory section, two transcription factors, UBF and SL1, have been characterized in in vitro Pol I transcription systems from vertebrates. The Rrn6/7 complex that we have characterized resembles SL1, but not UBF. UBF is a single polypeptide and has HMG box repeats with a strong DNA-binding activity, but Rrn6/7 is a heterotrimer and does not appear to have (strong) DNA-binding activity (D. Keys and M. Nomura, unpubl.). Although we do not know the amino acid sequence of $\mathrm{p} 66$, we failed to find any HMG-boxlike sequences in Rrn6p or Rrn7p. In contrast, there are some reasons to suspect that the Rrn6/7 complex might be related to SL1. First, in preliminary experiments, we prepared extracts from a strain in which the gene for TBP 
(SPT15) is replaced with an HAl epitope-tagged TBP gene. Preparations obtained by affinity binding of the (crude) D-300 fraction to the anti-HAl antibody, followed by elution with the HAl peptide, showed RRN6 and RRN7 activities, whereas control preparations with untagged extracts did not show such activity. This suggests that the Rrn6/7 complex might be associated with TBP directly or indirectly in crude extracts. (However, as is evident from the absence of TBP in the purified Rrn6/7 complex, such interactions, even if they are real, must be weak.) Second, SL1 purified from human cells contained three proteins (TAFs) with apparent molecular masses of 110,63 , and $48 \mathrm{kD}$ in addition to TBP /Comai et al. 1992). Similarly, SL1 (TIF-1B) purified from mouse was reported to contain three TAFs with apparent molecular masses of 95, 68, and $48 \mathrm{kD}$ (Eberhard et al. 1993). The sizes of these three TAF polypeptides resemble those of three proteins in the Rrn6/7 complex, which are 115, 66, and $56 \mathrm{kD}$ (values from SDS-PAGE analysis). Tjian and co-workers recently cloned cDNAs for the three TAFs contained in human SL1 (R. Tiian, pers. comm.), which has allowed direct sequence comparison between Rrn6p and $\mathrm{TAF}_{\mathrm{I}} 110$ and between $\mathrm{Rm} 7 \mathrm{p}$ and $\mathrm{TAF}_{\mathrm{I}} 48$. Sequence comparison by computer programs as well as visual inspection failed to find any significant sequence similarity between these two pairs of proteins. The leucine zipper-like motif in Rrn6p is not present in $\operatorname{TAF}_{\mathrm{I}} 110$, nor is the glutamine-rich region found in $\mathrm{Rrn} 7 \mathrm{p}$ present in $\mathrm{TAF}_{\mathrm{I}} 48$. Nevertheless, the absence of sequence similarity does not exclude the possible correspondence between the three TAFs in human SLl and the three proteins in the Rrn6/7 complex in S. cerevisiae. In Pol II systems, it was discovered recently that the essential yeast gene TSM1 has an obvious sequence similarity to a Drosophila TAF, $\mathrm{dTAF}_{\mathrm{II}} 150$, is associated with TBP in crude yeast extracts, and is therefore a yeast homolog of dTAF $_{\text {II }} 150$ (Verriizer et al. 1994). However, as is well recognized, evolutionary divergence of components appears to be much greater in Pol I than in Pol II transcription systems, perhaps reflecting the fact that Pol I is devoted to transcription of only a single essential gene, rDNA, and coevolution of rDNA promoters and transcription factors may have been easier compared with the Pol II system (Grummt et al. 1982; see also Bell et al. 1989|. Thus, the question of whether the three proteins in the Rrn6/7 complex correspond to TAFs in SL1 in vertebrates must await further studies.

Like SL1 in the human system (Bell et al. 1988), the Rrn6/7 complex alone or together with TBP failed to make a stable complex with the yeast rDNA promoter. Template commitment experiments have demonstrated that some essential factor(s) other than the Rrn6/7 complex is able to make a stable complex with template, which may be an intermediate in the pathway of initiation, and that the Rrn6/7 complex joins subsequently. Template commitment experiments were carried out previously with unfractionated yeast extracts, the effects of various deletion and linker-scanning mutations on the ability to sequester an essential factor(s) as well as their effects on the transcription activity were studied, and certain essential cis elements were defined in these respects (Kulkens et al. 1991). By the repetition of template commitment experiments similar to that shown in Figure 5 , but with various mutant templates as one of the two templates in competition, it should be possible to define-if there are (as we suspect)-DNA elements in the rDNA promoter that are responsible for stable binding of the Rrn6/7 complex to rDNA promoter.

The factor(s) that is present in rrn6 or $r r n 7$ mutant extracts and is responsible for the observed template commitment in the absence of the Rrn6/7 complex has not been identified. Like UBF in the vertebrate Pol I systems or like TFIIIA and TFIIIC in Pol III systems, the unidentified factor(s) may represent or include an assembly factor(s) that helps binding of essential factors including the Rrn6/7 complex. Extracts from other different rrn mutants show different kinds of partial or complete defects in the present in vitro transcription system. For example, the defect/s) in extracts from rrn3 mutants can be complemented by a protein fraction that can be separated from the Rrn6/7 complex in the heparin-agarose step shown in Figure 1 (R. Yamamoto, D. Keys, and M. Nomura, unpubl.). The availability of these mutant extracts as well as the corresponding $R R N$ genes should help to identify the factor(s) responsible for the initial template commitment and any other remaining essential factors.

Cellular contents of Rrn6p and Rrn7p appear to be very small compared with Pol I. By growing a strain carrying HAl-tagged $R R N 6$ in parallel with a strain carrying HA1-tagged RPA135, which encodes the second largest subunit of Pol I, we found that the cellular concentration of Rrn6p (and, hence, presumably Rrn $7 \mathrm{p}$ ) is $<1 \%$ of that of Pol I (in molar ratios) as judged by Western blot analysis with the anti-HAl antibody (R. Yamamoto, H. Wai, and $M$. Nomura, unpubl.). In contrast, the amounts of general transcription factors for Pol II in yeast cells appear to be higher. Koleske and Young (1994) reported the isolation of a Pol II holoenzyme that contains stoichiometric amounts of TFIIB, TFIIF, and TFIIH in addition to other factors such as SRB regulatory proteins. They estimated that the holoenzyme represents $6 \%$ of total Pol II; therefore, the amount of associated transcription factors are at least $6 \%$ of total Pol II. Specifically for TFIIB, they noted that a large amount of additional TFIIB exists, and its total amount is as high as $50 \%$ that of the total Pol II enzyme. Kornberg and co-workers also isolated a similar, but somewhat distinct, Pol II holoenzyme. This holoenzyme contained stoichiometric amounts of TFIIH and other mediator proteins, including SRB proteins, but not TFIIB or TFIIF, and the holoenzyme, and hence TFIIH, was reported to represent at least half of the total Pol II enzyme (Kim et al. 1994). A relatively small ratio of Rrn6/7 complex to Pol I might be sufficient for cellular needs if transcription factors (including the Rrn6/7 complex) were stably bound to the rRNA promoter region and were able to catalyze multiple Pol I initiation events. Regarding the reports on Pol II holoenzymes with many associated protein factors, we note that the Rrn6/7 complex in our extracts exists mostly as a separate entity 
from Pol I and, hence, appears to behave differently from the factors and SRB proteins associated with the polymerase in the yeast Pol II system.

Studies of rDNA transcription by Pol I by use of the yeast system have some distinct advantages. As is already evident from the discussion above, the in vivo significance of information obtained by in vitro experiments can be examined by genetic and physiological analysis. For example, by construction of strains with $R R N 6$ or RRN7 deletions whose growth is dependent on the expression of the GAL7-35S rDNA fusion on a plasmid, it has been demonstrated convincingly that both Rrn6p and Rrn7p are essential for Pol I function but are not required for Pol II or Pol III functions (this paper; L. $\mathrm{Vu}$ and M. Nomura, unpubl.; see also Nogi et al. 1991a). Because $\mathrm{TAF}_{\mathrm{I}}$ proteins in mammalian systems have been shown to differ from other known $\mathrm{TAF}_{\mathrm{II}}$ proteins (Comai et al. 1992; Eberhard et al. 1993), they are almost certainly Pol I specific, and all are probably essential for Pol I function. However, proof for being so in vivo is not an easy task in mammalian systems. Similarly, p66, a component of the Rrn6/7 complex discovered in the present study, is likely an essential component for the function of this complex and is probably unique to Pol I. However, formal proof for this inference must await isolation of the gene for this protein followed by appropriate genetic analyses.

\section{Materials and methods}

Media and buffers

YEP-glucose medium contains 1\% Bacto yeast extract (Difco),
$2 \%$ Bacto-peptone, and $2 \%$ glucose. YEP-galactose medium is the same as YEP-glucose medium except that $2 \%$ galactose is substituted for glucose. Buffer A is $20 \mathrm{~mm}$ Tris-acetate ( $\mathrm{pH} 8.0)$, $10 \mathrm{~mm} \mathrm{Mg}$ acetate, $0.2 \mathrm{~mm}$ EDTA, $10 \%$ glycerol, $10 \mathrm{~mm} \beta$-mercaptoethanol, and $0.1 \mathrm{~mm}$ phenylmethylsulfonyl flouride (PMSF). Buffer E is $20 \mathrm{~mm}$ Tris-acetate (pH 8.0), $10 \mathrm{~mm} \mathrm{Mg}$ acetate, $0.2 \mathrm{~mm}$ EDTA, $10 \%$ glycerol, $200 \mathrm{~mm} \mathrm{~K}$ glutamate, 0.1 mM PMSF, and $0.5 \mathrm{mM}$ dithiothreitol. Buffer $\mathrm{S}$ is $200 \mathrm{mM}$ Trisacetate ( $\mathrm{pH} 8.0$ ), 10\% glycerol, $10 \mathrm{mM} \mathrm{Mg}$ acetate, $10 \mathrm{~mm} \beta$-mercaptoethanol, and $1 \mathrm{mM}$ PMSF. Buffer TA is $20 \mathrm{~mm}$ Tris-acetate (pH 8.0), $0.2 \mathrm{~mm}$ EDTA, $10 \mathrm{~mm} \mathrm{Mg}$ acetate, $10 \mathrm{~mm} \beta$-mercaptoethanol, $1 \mathrm{~mm}$ PMSF, $5 \mathrm{~mm}$ benzamidine- $\mathrm{HCl}, 0.1 \% \mathrm{~N}$-octyl glucoside, $0.2 \mathrm{mg} / \mathrm{ml}$ of aprotinin, and $10 \mu \mathrm{g} / \mathrm{ml}$ of the following protease inhibitors: pepstatin, bestatin, aprotinin, chymostatin, leupeptin, and antipain. Buffer TA was supplemented with several different concentrations of $\mathrm{KCl}$ and glycerol, and these concentrations are given by numbers following TA. Thus, buffer TA $300 / 30$ is buffer TA supplemented with $300 \mathrm{~mm} \mathrm{KCl}$ and $30 \%$ glycerol.

\section{Strains and plasmids}

The strains and plasmids used in this study are listed in Table 1. Some essential procedures used to construct plasmids and strains are described below in connection with HAl epitope tagging and gene disruption.

\section{Cloning and sequencing of RRN6 and RRN7}

Strain NOY630 carrying the rrn6-1 mutation (isolation number 195) and strain NOY629 carrying the rrn 7-1 mutation (isolation number 801), both of which cannot grow on glucose, were used to clone RRN6 and RRN7 genes, respectively. The yeast genomic library (Nakayama et al. 1985) carrying chromosomal DNA inserted at the BamHI site of YCpN1 vector (CEN3 ARS1 TRP1) was used to isolate transformants that were able to grow on

Table 1. Yeast strains and plasmids used

\begin{tabular}{|c|c|}
\hline Strain or plasmid & Description \\
\hline \multicolumn{2}{|l|}{ Strains } \\
\hline JHRY20-2C $\Delta 1$ & MATa his3-4200 ura3-52 leu2-3,112 pep4- $\triangle 1:: U R A 3$ \\
\hline (NOY203) & (Ammerer et al. 1986) \\
\hline NOY397 & MATa/ $\alpha$ ade2-1/ade2-1 ura3-1/ura3-1 his3-11/his3-11 trpl-1/trp1-1 leu2-3,112/leu2-3,112 can1-100/can1-100 \\
\hline NOY418 & MATa ade2 ade3 leu2 ura3 lys2 can1 pNOY103 \\
\hline NOY552 & MATa ade2-1 ura3-1 leu2-3,112 trp1-1 his3-11 can1-100 rrn7::LEU2 pNOY209 \\
\hline NOY558 & MAT $\alpha$ ade2-1 ura3-1 leu2-3,112 trp1-1 his3-11 can1-100 rrn7::LEU2 pNOY103 \\
\hline NOY 567 & MAT $\alpha$ ade2-1 ura3-1 lu2-3,112 trp1-1 his3-11 can1-100 rrn6::LEU2 pNOY103 \\
\hline NOY573 & MAT $\alpha$ ade2-1 ura3-1 leu2-3,112 trp-1 his3-11 can1-100 rrn6::HIS3 pNOY214 \\
\hline NOY 628 & MATa ade2 ade3 ura3 leu2 lys2 can1 rm6-2 pNOY103 (rrn6 mutant 793; Nogi et al. 1991a) \\
\hline NOY 629 & 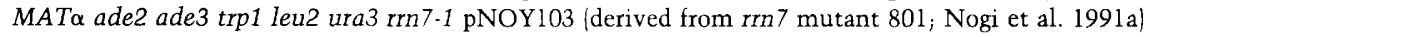 \\
\hline NOY 630 & MAT $\alpha$ ade2 ade3 trp1 leu2 ura3 rrn6-1 pNOY103 (derived from rm6 mutant 195; Nogi et al. 1991a) \\
\hline \multicolumn{2}{|r|}{ 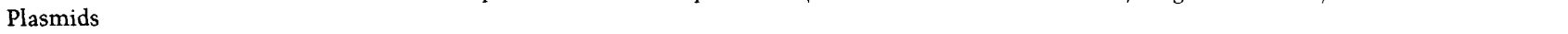 } \\
\hline pRS315 & $\begin{array}{l}\text { Escherichia coli-yeast shuttle vector carrying CEN6 ARSH4 LEU2 and with multicloning site from pBluescript KS } \mid- \text { ) } \\
\text { (Sikorski and Hieter 1989) }\end{array}$ \\
\hline pRS316 & $\begin{array}{l}\text { E. coli-yeast shuttle vector carrying CEN6 ARSH4 URA3 and with multicloning site from pBluescript KS(- / (Sikorski and } \\
\text { Hieter 1989) }\end{array}$ \\
\hline pNOY103 & High-copy-number plasmid carrying GAL7-35S rDNA, $A D E 3,2 \mu$ amp (Nogi et al., 1991a) \\
\hline pNOY204 & A derivative of pSR315 with 3.6-kb HindIII fragment carrying RRN6 inserted into the HindIII site (see Fig. la) \\
\hline pNOY209 & $\begin{array}{l}\text { A derivative of PRS3 } 16 \text { with the } 2.5-\mathrm{kb} S p h \mathrm{I}-X b a \mathrm{I} \text { fragment carrying HAl-epitope-tagged } R R N 7 \text { inserted between } X h o I \text { and } \\
X b a I \text { sites }\end{array}$ \\
\hline pNOY214 & A derivative of pRS315 with the $3.7-\mathrm{kb}$ HindIII fragment carrying HAl-epitope-tagged $R R N 6$ inserted into the HindIII site \\
\hline pNOY265 & $\begin{array}{l}\text { A derivative of pRS316 with the } 2.5-\mathrm{kb} S p h \mathrm{I}-X b a \mathrm{I} \text { fragment carrying } R R N 7 \text { inserted between } X h o I \text { and } X b a I \text { sites (see } \\
\text { Fig. } 1 \mathrm{c} \text { ) }\end{array}$ \\
\hline pNOY3166 & A derivative of pBluescriptII KS $|-|$ with a fragment carrying $r m 7:: L E U 2$ between $X$ hoI and $X b a I$ sites (see text and Fig. $1 \mathrm{~d}$ ) \\
\hline pNOY3193 & pUC19 derivative with a fragment carrying HAl-epitope-tagged $r m 6:: H I S 3$ inserted at the HindIII site (see text and Fig. $1 \mathrm{~b}$ ) \\
\hline pSIRT & A derivative of pEMBL Ye30- $\Delta 6$ that carries the mini rDNA gene called SIRT (Musters et al. 1989) \\
\hline pSIRT $\Delta+27 /+128$ & A derivative of pSIRT. DNA between +27 and +128 is deleted (Musters et al. 1989) \\
\hline
\end{tabular}


glucose, as described previously (Nogi et al. 1993). Plasmids were recovered and subcloning was carried out to localize RRN6 and RRN7 genes. The structure of the DNA insert in pNOY204 carrying $R R N 6$ and that in pNOY265 carrying $R R N 7$ are shown in Figure 1, a and c, respectively. The 3.6-kb HindIII fragment in pNOY204 and the 2.46-kb SphI-XbaI fragment in pNOY265 were further subcloned into pUC18 and pUC19, and their DNA sequences were determined by the dideoxy method with the Tag DyeDeoxy Terminator Cycle Sequencing Kit (Applied Biosystems, Foster City, CA).

\section{Construction of genes encoding HA1-epitope- tagged Rrn6p and Rrn7p}

The HAl epitope from influenza virus (Wilson et al. 1984), YPYDVPDYA, was attached to the amino terminus of Rrn6p and Rrn7p. For RRN6, the 39-bp oligonucleotide, CGG CAT ATG TAC CCA TAC GAC GTC CCA GAC TAC GCT AGC ।ATG is the new start codon), which encodes MYPYDVPDYAS, was inserted immediately in front of the ATG start codon of RRN6 by oligonucleotide-directed mutagenesis (Kontis and Goldin 1993) with a derivative of M13mpl 8 carrying the 924-bp HindIII-KpnI fragment of $R R N 6$ as template and a 70-nucleotide oligonucleotide containing the above 39 -nucleotide sequence flanked by $R R N 6$ sequences as a mutagenesis primer. Candidate mutant phages were isolated, and the mutational alteration was confirmed by DNA sequencing. The new HindIII-KpnI fragment (963 bp) was subcloned into pUC19, and the missing RRN6coding region was introduced to restore the complete $R R N 6$ gene with the HA1 epitope (pNOY3167). The 3.68 -kb HindIII fragment carrying HA1-RRN6 was then isolated from this plasmid and inserted into the HindIII site of pRS315, leading to pNOY214.

For RRN7, the 30-bp oligonucleotide, ATG TAC CCA TAC GAT GTT CCA GAT TAC GCT (ATG is the new start codon), which encodes MYPYDVPDYA, was inserted immediately in front of the ATG start codon by a polymerase chain reaction (PCR) method with pNOY3190, which carries the 769-bp HindIII-BamHI fragment containing the RRN7 start site, as template. One primer was the 72-nucleotide oligonucleotide containing the above 30 nucleotide sequence flanked by RRN7 sequences, and the other was a M13/pUC sequencing primer outside the HindIII-BamHI insert. The PCR product was digested with $\mathrm{XbaI}$ and $\mathrm{KpnI}$, the fragment containing the inserted HAl-tagged coding sequence was recovered by cloning, and the mutational alteration was confirmed by DNA sequencing. The 331-bp HindIII-AatII fragment of pNOY265, which carries RRN7 in pRS316, was replaced by the corresponding HindIIIAatII fragment with the 30-bp insert, yielding pNOY209 carrying HAl-RRN7.

\section{Construction of $\mathrm{rm} 6$ and $\mathrm{rrn} 7$ deletion mutants}

pNOY3167 carrying HA1-RRN6 (see above) was digested with $B a m H I$, and 2144 bp of the RRN6-coding region was replaced by a $0.9-\mathrm{kb}$ BamHI fragment carrying HIS3 (see Fig. lb). The resulting plasmid (pNOY3193) was digested with NarI and ClaI, the NarI-ClaI fragment carrying rrn6::HIS3 was transformed into a diploid strain (NOY397), and $\mathrm{His}^{+}$transformants were selected. One of the transformants, NOY569, was confirmed by tetrad analysis to carry the expected rrn6::HIS3 on one chromosome and was used to construct a haploid strain (NOY573) carrying $r m 6:: H I S 3$ on the chromosome and the HA1-RRN6 gene on a plasmid (pNOY214) as well as a haploid strain (NOY567) carrying rrn6::HIS3 on the chromosome and the pGAL7-35S rDNA on a plasmid (pNOY103).
To construct a similar $r m 7$ deletion mutant, pNOY3165 carrying $R R N 7$ on the $2.46-\mathrm{kb}$ SphI-XbaI fragment was digested with BamHI (see Fig. 1c), and the 378 bp of the RRN7-coding region was replaced by a $3.1-\mathrm{kb} B g l I$ fragment carrying $L E U 2$ (see Fig. 1d). The resulting plasmid (pNOY3166) was digested with $H$ indIII and $\mathrm{XbaI}$, and the $4.6-\mathrm{kb}$ fragment carrying most of rrn $7:: L E U 2$ was isolated. This fragment was transformed into a diploid strain NOY397 with selection for Leu2 ${ }^{+}$. One of the transformants, NOY548, was confirmed by tetrad analysis to carry $\operatorname{rrn} 7:: L E U 2$ on one chromosome and was used to construct a haploid strain (NOY552) expressing only HAl-RRN7 from a plasmid (pNOY209) and a haploid strain (NOY558) carrying pNOY103 that allows galactose-dependent growth without intact RRN7.

\section{Preparation of transcription extracts}

Cells were grown in YEP-glucose ffor wild-type cultures; strain JHRY20-2C $\Delta 1$ or strains such as NOY573 and NOY552 expressing HAl-tagged proteins) or YEP-galactose (for mutant strains, such as NOY567 and NOY558, whose growth requires the presence of galactose) at $30^{\circ} \mathrm{C}$ to an $A_{600}$ of 0.9 or less. Cultures were poured onto ice immediately, and cells were harvested by centrifugation. This and all subsequent steps were performed at $4^{\circ} \mathrm{C}$. The cell pellet was washed once in cold distilled $\mathrm{H}_{2} \mathrm{O}$, resuspended in buffer $\mathrm{S}$, and stored at $-70^{\circ} \mathrm{C}$. For preparation of extracts, a thawed cell suspension containing 10-15 grams of cells was pelleted by centrifugation. The cell pellets were resuspended in buffer S supplemented with $400 \mathrm{~mm}$ ammonium sulfate $(\mathrm{pH} 8.0)$, in a final volume of $30 \mathrm{ml}$. Cells were pelleted again by centrifugation, and the pellets were homogenized by grinding with mortar and pestle under liquid nitrogen as described by Schultz et al. (1991). The homogenized paste was resuspended in $9 \mathrm{ml}$ of buffer $\mathrm{S}(0.1 \mathrm{mM}$ PMSF instead of $1 \mathrm{mM})$ and clarified by centrifugation at $10,000 \mathrm{rpm}$ for $10 \mathrm{~min}$. The extracts were then diluted to $40 \mathrm{ml}$ with buffer S (0.1 mM PMSF) and centrifuged at $65,000 \mathrm{rpm}$ for $60 \mathrm{~min}$ in a Beckman $80 \mathrm{Ti}$ ultracentrifuge rotor. The supernatant (S100) was carefully removed with a syringe fitted with teflon tubing, taking care to avoid the flaky white layer at the top of the tubes, removing approximately two-thirds of the supernatant overall. The extracts were dialyzed against 2 liters of buffer A for $3 \mathrm{hr}$. The conductivity was then adjusted to that of $120 \mathrm{mM} \mathrm{KCl}$ in buffer $A$ by addition of either buffer A without salt or $1 \mathrm{M} \mathrm{KCl}$ in buffer A. The dialyzed extract was then centrifuged at $10,000 \mathrm{rpm}$ for $10 \mathrm{~min}$, and the supernatant was loaded onto a phosphocellulose column (phosphocellulose, fibrous form, Sigma, St. Louis, $\mathrm{MO}$; with an $\sim 10-\mathrm{ml}$ final bed volume) equilibrated in $120 \mathrm{~mm}$ $\mathrm{KCl}$ in buffer $\mathrm{A}$. The flowthrough from this column was loaded directly onto a DEAE column (Whatman DE52, Whatman, Maidstone, England; with an $\sim 5-\mathrm{ml}$ final bed volume) equilibrated in $120 \mathrm{mM} \mathrm{KCl}$ in buffer A. Both columns were washed with 4 column volumes of $120 \mathrm{~mm} \mathrm{KCl}$ in buffer $\mathrm{A}$ and eluted with $300 \mathrm{mM} \mathrm{KCl}$ in buffer A. Peak protein fractions (as monitored by absorbance at $280 \mathrm{~nm}$ ) were pooled and dialyzed against buffer $E$. The dialyzed preparation obtained from the DEAE column (D-300 fraction) typically contained $\sim 3-6 \mathrm{mg} / \mathrm{ml}$ protein; the preparation eluted from the phosphocellulose column with $300 \mathrm{mM} \mathrm{KCl}$ (PC-300 fraction) contained $\sim 0.2-0.4 \mathrm{mg} / \mathrm{ml}$ of protein.

\section{Fractionation of RRN6 and RRN7 activities}

For large-scale preparation of transcription extracts, wild-type cells were grown at $30^{\circ} \mathrm{C}$ in 16.5 liters of YEP-glucose in a fermentor to an $A_{600}$ of 0.9 . Cells were harvested, washed with 
$\mathrm{H}_{2} \mathrm{O}$, resuspended in buffer $\mathrm{S}$, and stored at $-70^{\circ} \mathrm{C}$ as described above. Approximately 100-140 grams of cells 12 fermentor batches) was resuspended in $\sim 150 \mathrm{ml}$ of buffer $\mathrm{S}$ plus $35 \mathrm{ml}$ of $4 \mathrm{M}$ ammonium sulfate (pH 8.0$)$, plus $150 \mathrm{ml}$ of acid-washed glass beads (0.4- to $0.5-\mathrm{mm}$ diam.) and transferred to a $400-\mathrm{ml}$ metal Bead-beater chamber (Biospec Products, Bartlesville, OK). Buffer $S$ was added to fill the chamber, bringing the final volume of liquid to $350 \mathrm{ml}$, and the cells were homogenized in the Bead-beater apparatus, kept in an ethanol-ice bath, at a reduced voltage (95 V) with 30 pulses of $30 \mathrm{sec}$ with a $90-\mathrm{sec}$ pause between each pulse. The homogenate was clarified by centrifugation at $10,000 \mathrm{rpm}$ for $10 \mathrm{~min}$, followed by centrifugation at $45,000 \mathrm{rpm}$ for $60 \mathrm{~min}$ in a Beckman $60 \mathrm{Ti}$ rotor. The supernatant $(\mathrm{S} 100)$ was removed and dialyzed, and its $\mathrm{KCl}$ concentration was adjusted to $120 \mathrm{mM}$, as described in the previous section. The extract was then loaded onto a phosphocellulose column (with an $\sim 90-\mathrm{ml}$ final bed volume) equilibrated in $120 \mathrm{~mm}$ $\mathrm{KCl}$ in buffer $\mathrm{A}$. The flowthrough fraction was loaded directly onto a DEAE column (Whatman DE52; 45- to 50-ml final bed volume), the column was washed, and elution was done with $300 \mathrm{mM} \mathrm{KCl}$ in buffer A as described above. Protein-containing fractions were pooled and loaded directly onto a heparin-agarose column (5-ml cartridge; Bio-Rad, Hercules, CA) equilibrated in $300 \mathrm{~mm} \mathrm{KCl}$ in buffer A. After washing with $300 \mathrm{mM} \mathrm{KCl}$ in buffer A, elution was done with $400 \mathrm{~mm} \mathrm{KCl}$ in buffer $\mathrm{A}$. The protein peak (H-400 fraction) was concentrated by Centricon- 10 (Amicon, Berkeley, CA) and loaded onto a Superose 6 (Pharmacia, Piscataway, NI/ gel filtration column equilibrated in buffer $\mathrm{E}$, and protein fractionation by size was carried out with the same buffer.

\section{Immunoaffinity purification of an Rrn6/7 complex}

Monoclonal antibody $12 \mathrm{CA} 5$ specific to the HA1 epitope and the epitope peptide (YPYDVPDYA) were obtained from Berkeley Antibody Co. (Richmond, CA). Cross-linking of this antibody, purified as IgG, to protein A-Sepharose or protein G-Sepharose (both from Pharmacia) was carried out with dimethyl pimelimidate according to the protocol provided by a supplier of the reagent. Extracts were prepared from three strains expressing HA1-Rrn6p (NOY573), HAl-Rrn7p (NOY552) or without HAl epitope tagging (NOY418) as described in the previous section except that cell breakage was done in buffer $\mathrm{S}$ supplemented with NP-40 $(0.1 \%)$, benzamidine $\mathrm{HCl}(5.5 \mathrm{~mm})$, ammonium sulfate $(400 \mathrm{~mm})$, and each of the following protease inhibitors at $10 \mu \mathrm{g} / \mathrm{ml}$ : aprotinin, chymostatin, leupeptin, antipain, bestatin, and pepstatin. Supernatants obtained after centrifugation at $30,000 \mathrm{rpm}$ for $30 \mathrm{~min}$ in Beckman type 35 rotor were used for affinity purification. About $200-\mathrm{ml}$ supernatants derived from $\sim 120$ grams of cells were mixed with $5 \mathrm{ml}$ of protein A-Sepharose beads carrying cross-linked 12CA5 antibodies $(\sim 20 \mathrm{mg}$ of $\mathrm{IgG} / \mathrm{ml})$ in a bottle, and rolled gently on a roller drum at $4^{\circ} \mathrm{C}$ for $4 \mathrm{hr}$. The antibody beads were recovered by centrifugation, transferred to a column, washed five times with $5 \mathrm{ml}$ of TA500/30 buffer, three times with $5 \mathrm{ml}$ of TA300/ 30 buffer, and then transferred to a centrifuge tube with $10 \mathrm{ml}$ of TA300/30 buffer. The antibody beads were recovered by centrifugation, mixed with $5 \mathrm{ml}$ of $12 \mathrm{CA} 5$ peptide $(1 \mathrm{mg} / \mathrm{ml}$; in TA300/30 buffer), and left for $30 \mathrm{~min}$ at room temperature to elute complexes containing HA1-Rrn6p or HA1-Rrn7p. The antibody beads were recovered by centrifugation, and the elution step was repeated. Eluates were combined and applied to a small heparin-Sepharose CL6B column (Pharmacia; 0.6-ml bed volume) equilibrated with TA300/30 buffer. The column was washed five times with $1-\mathrm{ml}$ aliquots of TA300/30 buffer, followed by elution of the Rrn6/7 complex with TA600/50 buffer
(0.6 $\mathrm{ml}$ each, five to six times). Then $0.6-\mathrm{ml}$ eluate fractions were collected, and each fraction was assayed for activity to complement rrn6 mutant extracts. Three to four fractions that showed a high complementation activity were combined and mixed with an equal volume of TA0/10 buffer to make the final buffer composition equal to TA300/30. The resultant solution (about $5 \mathrm{ml}$ ) was mixed with the $12 \mathrm{CA} 5$ antibody cross-linked to protein $\mathrm{G}-$ Sepharose beads $\sim \sim 150 \mu$ l of beads prewashed with TA300/30 buffer) and rolled gently at $4^{\circ} \mathrm{C}$ for $4 \mathrm{hr}$ as described above. After washing the beads with $1 \mathrm{ml}$ of TA300/30 3 times, Rrn6/7 complexes were eluted with $175 \mu$ l of $12 \mathrm{CA} 5$ peptide (1 $\mathrm{mg} / \mathrm{ml}$ twice. The eluates were combined and kept frozen at $-70^{\circ} \mathrm{C}$

\section{Transcription assays}

In vitro reactions were performed as described previously (Riggs and Nomura 1990), with minor modifications. The DNA template used for most assays is pSIRT, which carries the SIRT yeast rDNA minigene as described previously (Kulkens et al. 1991; Musters et al. 1989|. Unlike the transcription extract used previously (Kulkens et al. 1991), which with this template generated a 681-nucleotide transcript that was processed at the $3^{\prime}$ end of the minigene, the fractionated transcription extract used in this study generated an $\sim 765$-nucleotide transcript that is not processed, but whose $3^{\prime}$ end is apparently generated by termination at the proper site (Lang and Reeder 1993). Template B in Figures 5 and 6 is a derivative (pSIRT $\Delta+27 /+128$ ) of pSIRT carrying a 100-bp deletion downstream from the promoter (Musters et al. 1989), producing a specific transcript of $\sim 665$ nucleotides. The standard $40-\mu \mathrm{l}$ reaction mixture contained 20 mM Tris-acetate $(\mathrm{pH} 8.0), 8 \mathrm{~mm} \mathrm{Mg}$ acetate, $0.5 \mathrm{~mm}$ dithiothreitol, $175 \mathrm{~mm} \mathrm{~K}$ glutamate, $200 \mu \mathrm{m}$ ATP, $200 \mu \mathrm{M}$ UTP, $200 \mu \mathrm{M}$ CTP, $15 \mu \mathrm{M}$ GTP, $10 \mu \mathrm{Ci}\left[\alpha^{-}{ }^{32} \mathrm{P}\right]-\mathrm{GTP}(3000 \mathrm{Ci} / \mathrm{mmole}$; New England Nuclear Biochemicals), 200 ng of DNA template as described above, and protein fractions. Typical in vitro reactions contained $3-6 \mu$ l of D-300 fraction $(\sim 20 \mu \mathrm{g}$ of protein) plus $4-10 \mu 1$ of PC-300 fraction ( 2-4 $\mu$ g of protein). These fractions were preincubated with DNA template in the complete reaction mixture except for the nucleoside triphosphates for 10-15 min at room temperature $\left(\sim 25^{\circ} \mathrm{C}\right)$. Transcription was initiated by addition of nucleoside triphosphates. Reactions were incubated for $40 \mathrm{~min}$ at room temperature. The reaction was then stopped by the addition of EDTA, and RNA was recovered and analyzed by $5 \%$ urea-PAGE as described previously (Riggs and Nomura 1990). For all of the experiments described in this paper, mutant extracts were prepared from gene-disrupted strains (NOY558 and NOY567). For some earlier experiments, extracts were prepared from point mutants (NOY628 and NOY629). No difference was observed between these two sets of extracts.

\section{Other materials and methods}

Separation of proteins by SDS-PAGE followed by Western immunoblot analysis was carried out as described previously (Nogi et al. 1993), and an immunoassay kit (Bio-Rad Laboratories, Richmond, CA) was used for antibody visualization. Silver staining of proteins after SDS-PAGE was carried out by use of a kit from Bio-Rad according to the protocol provided by the supplier. Purified yeast TBP (Horikoshi et al. 1989) was a kind gift from Drs. M. Horikoshi and R.G. Roeder (Rockefeller University, NY). Rabbit polyclonal antibodies against yeast TBP were generous gifts from Dr. A.J. Berk (University of California, Los Angeles) and from Drs. D. Poon and P.A. Weil (Vanderbilt University, Nashville, TN). Rabbit polyclonal antibodies against Rrn6p were prepared in this laboratory by construction of an 
expression plasmid carrying a GST-RRN6 fusion gene encoding a part of Rrn6p (from amino acid 42 to 666 ) fused to glutathione$S$-transferase in pGEX3X (Pharmacia), expressing the gene in Escherichia coli and injecting the purified fusion protein into rabbits. The polyclonal antibody preparation obtained in this way gave several cross-reacting bands in crude extracts and was used only to confirm the presence of Rrn6p in the affinity-purified Rrn6/7 complex containing HAl epitope-tagged Rrn7p.

\section{Acknowledgments}

We thank Drs. R.G. Roeder for providing purified TBP, and A.J. Berk and P.A. Weil for anti-TBP antibodies. We also thank Dr. $\mathrm{R}$. Tjian for providing unpublished results on TAFs in SLl, and Drs. S. Sandmeyer and J. Keener for critical reading of the manuscript. This work was supported by U.S. Public Health grant R37GM35949 from the National Institutes of Health.

The publication costs of this article were defrayed in part by payment of page charges. This article must therefore be hereby marked "advertisement" in accordance with 18 USC section 1734 solely to indicate this fact.

\section{References}

Ammerer, G., C.P. Hunter, J.H. Rothman, G.C. Saari, L.A. Valls, and T.H. Stevens. 1986. PEP4 gene of Saccharomyces cerevisiae encodes proteinase A, a vacuolar enzyme required for processing of vacuolar precursors. Mol. Cell. Biol. 6: 24902499.

Bachvarov, D. and T. Moss. 1991. The RNA polymerase I transcription factor xUBF contains 5 tandemly repeated HMG homology boxes. Nucleic Acids Res. 19: 2331-2335.

Bell, S.P., R.M. Learned, H.-M. Jantzen, and R. Tjian. 1988. Functional cooperativity between transcription factors UBF1 and SL1 mediates human ribosomal RNA synthesis. Science 241: 1192-1197.

Bell, S.P., C.S. Pikaard, R.H. Reeder, and R. Tjian. 1989. Molecular mechanisms governing species-specific transcription of ribosomal RNA. Cell 59: 489-497.

Comai, L., N. Tanese, and R. Tjian. 1992. The TATA-binding protein and associated factors are integral components of the RNA polymerase I transcription factor, SL1. Cell 68: 965976.

Cormack, B.P. and K. Struhl. 1992. The TATA-binding protein is required for transcription by all three nuclear RNA polymerases in yeast cells. Cell 69: 685-696.

Courey, A.J. and R. Tjian. 1988. Analysis of Spl in vivo reveals multiple transcriptional domains, including a novel glutamine-rich activation motif. Cell 55: 887-898.

Eberhard, D., L. Tora, J.-M. Egly, and I. Grummt. 1993. A TBPcontaining multiprotein complex (TIB-IB) mediates transcription specificity of murine RNA polymerase I. Nucleic Acids Res. 21: 4180-4186.

Elledge, S.J. and R.W. Davis. 1987. Identification and isolation of the gene encoding the small subunit of ribonucleotide reductase from Saccharomyces cerevisiae: DNA damage-inducible gene required for mitotic viability. Mol. Cell. Biol. 7: 2783-2793.

Grummt, I., E. Roth, and M.R. Paule. 1982. Ribosomal RNA transcription in vitro is species specific. Nature 296: 173175.

Hernandez, N. 1993. TBP, a universal eukaryotic transcription factor? Genes \& Dev. 7: 1291-1308.

Hisatake, K., T. Nishimura, Y. Maeda, K. Hanada, C.-Z. Song, and M. Muramatsu. 1991. Cloning and structural analysis of
cDNA and the gene for mouse transcription factor UBF. Nucleic Acids Res. 19: 4631-4637.

Horikoshi, M., C.K. Wang, H. Fujii, J.A. Cromlish, P.A. Weil, and R.G. Roeder. 1989. Purification of a yeast TATA boxbinding protein that exhibits human transcription factor IID activity. Proc. Natl. Acad. Sci. 86: 4843-4847.

Jantzen, H.-M., A. Admon, S.P. Bell, and R. Tjian. 1990. Nucleolar transcription factor hUBF contains a DNA-binding motif with homology to HMG proteins. Nature 344: 830-836.

Kim, Y.-J., S. Bjorklund, Y. Li, M.H. Sayre, and R.D. Kornberg. 1994. A multiprotein mediator of transcriptional activation and its interaction with the C-terminal repeat domain of RNA polymerase II. Cell 77: 599-608.

Koleske, A.J. and R.A. Young. 1994. An RNA polymerase II holoenzyme responsive to activators. Nature 368: 466-469.

Kontis, K.J. and A.L. Goldin. 1993. Site-directed mutagenesis of the putative pore region of the rat IIA sodium channel. Mol. Pharmacol. 43: 635-644.

Kulkens, T., D.L. Riggs, J.D. Heck, R.J. Planta, and M. Nomura. 1991. The yeast RNA polymerase I promoter: Ribosomal DNA sequences involved in transcription initiation and complex formation in vitro. Nucleic Acids Res. 19: 53635370 .

Lang, W.H. and R.H. Reeder. 1993. The REB1 site is an essential component of a terminator for RNA polymerase I in Saccharomyces cerevisiae. Mol. Cell. Biol. 13: 649-658.

Lue, N.F. and R.D. Kornberg. 1990. Accurately initiated, enhancer-dependent transcription by RNA polymerase I in yeast extracts. I. Biol. Chem. 265: 18091-18094.

McStay, B., C.-H. Hu, C.S. Pikaard, and R.H. Reeder. 1991. $\mathrm{xUBF}$ and Ribl are both required for formation of a stable polymerase I promoter complex in X. laevis. EMBO $I$. 10: 2297-2303.

Moss, T. and V.Y. Stefanovsky. 1994. Promotion and regulation of ribosomal transcription in eukaryotes by RNA polymerase I. Prog. Nucleic Acids Res. Mol. Biol. (in press).

Musters, W., J. Knol, P. Maas, A.F. Dekker, H. van Heerikhuizen, and R.J. Planta. 1989. Linker scanning of the yeast RNA polymerase I promoter. Nucleic Acids Res. 17: 9661-9678.

Nakayama, N., A. Miyajima, and K. Arai. 1985. Nucleotide sequences of STE2 and STE3, cell type-specific sterile genes from Saccharomyces cerevisiae. EMBO J. 4: 2643-2648.

Nogi, Y., L. Vu, and M. Nomura. 1991a. An approach for isolation of mutants defective in $35 \mathrm{~S}$ ribosomal RNA synthesis in Saccharomyces cerevisiae. Proc. Natl. Acad. Sci. 88: 70267030.

Nogi, Y., R. Yano, and M. Nomura. 1991b. Synthesis of large rRNAs by RNA polymerase II in mutants of Saccharomyces cerevisiae defective in RNA polymerase I. Proc. Natl. Acad. Sci. 88: 3962-3966.

Nogi, Y., R. Yano, J. Dodd, C. Carles, and M. Nomura. 1993. Gene RRN4 in Saccharomyces cerevisiae encodes the A12.2 subunit of RNA polymerase I and is essential only at high temperatures. Mol. Cell. Biol. 13: 114-122.

Nomura, M., Y. Nogi, R. Yano, M. Oakes, D.A. Keys, L. Vu, and J.A. Dodd. 1993. RNA Polymerase I, the nucleolus of $35 \mathrm{~S}$ rRNA in the yeast Saccharomyces cerevisiae. In The translational apparatus (ed. K. H. Nierhaus, F. Franceschi, and A.R. Subramanian|, pp. 89-99. Plenum Press, New York.

O'Mahony, D.J. and L.I. Rothblum. 1991. Identification of two forms of the RNA polymerase I transcription factor UBF. Proc. Natl. Acad. Sci. 88: 3180-3184.

Paule, M.R. 1994. Transcription of ribosomal RNA by eukaryotic RNA polymerase I. In Transcription: Mechanisms and regulation (ed. R.C. Conaway and J.W. Conaway), pp 83-106. Raven Press, New York. 


\section{Keys et al.}

Pugh, B.F. and R. Tjian. 1992. Diverse transcriptional functions of the multisubunit eukaryotic TFIID complex. 7 . Biol. Chem. 267: 679-682.

Radebaugh, C.A., J.L. Matthews, G.K. Geiss, F. Liu, J.-M. Wong, E. Bateman, S. Camier, A. Sentenac, and M.R. Paule. TATA box-binding protein $(\mathrm{TBP})$ is a constituent of the polymerase I-specific transcription initiation factor TIF-IB (SL1) bound to the rRNA promoter and shows differential sensitivity to TBP-directed reagents in polymerase I, II, and III transcription factors. Mol. Cell. Biol. 14: 597-605.

Reeder, R.H. 1992. Regulation of transcription by RNA polymerase I. In Transcriptional regulation, (ed. S.L. McKnight and K.R. Yamamoto), vol. 2, pp. 315-348. Cold Spring Harbor Laboratory Press, Cold Spring Harbor, New York.

Riggs, D.L. and M. Nomura. 1990. Specific transcription of Saccharomyces cerevisiae $35 \mathrm{~S}$ rDNA by RNA polymerase I in vitro. J. Biol. Chem. 265: 7596-7603.

Schnapp, A. and I. Grummt. 1991. Transcription complex formation at the mouse rDNA promoter involves the stepwise association of four transcription factors and RNA polymerase I. I. Biol. Chem. 266: 24588-24595.

Schultz, M.C., S.Y. Choe, and R.H. Reeder. 1991. Specific initiation by RNA polymerase I in a whole-cell extract from yeast. Proc. Natl. Acad. Sci. 88: 1004-1008.

Sikorski, R.S. and P. Hieter. 1989. A system of shuttle vectors and yeast host strains designed for efficient manipulation of DNA in Saccharomyces cerevisiae. Genetics 122: 19-27.

Skala, J., L.Van Dyck, B. Purnelle, and A. Goffeau. 1992. The sequence of an $8 \mathrm{~kb}$ segment on the left arm of chromosome II from Saccharomyces cerevisiae identifies five new open reading frames of unknown functions, two tRNA genes and two transposable elements. Yeast 8: 777-785.

Smith, S.D., E. Oriahi, D. Lowe, H.-F. Yang-Yen, D. O'Mahony, K. Rose, K. Chen, and L.I. Rothblum. 1990. Characterization of factors that direct transcription of rat ribosomal DNA. Mol. Cell. Biol. 10: 3105-3116.

Smith, S.D., D.J. O'Mahony, B.T. Kinsella, and L.I. Rothblum. 1993. Transcription from the rat $45 \mathrm{~S}$ ribosomal DNA promoter does not require the factor UBF. Gene Expression 3: 229-236.

Sollner-Webb, B. and E.B. Mougey. 1991. News from the nucleolus: rRNA gene expression. Trends Biochem. Sci. 16: 58 62.

Thuriaux, P. and A. Sentenac. 1992. Yeast nuclear RNA polymerases, I. In The molecular and cellular biology of the yeast Saccharomyces cerevisiae: Gene expression (ed. E.W. Jones, J.R. Pringle, and J.R. Broach), pp 1-48. Cold Spring Harbor Press, Cold Spring Harbor, New York.

Verrijzer, C.P., K. Yokomori, J.-L. Chen, and R. Tjian. 1994. Drosophila TAF $_{\text {II }}$ 150: Similarity to yeast gene TSM-1 and specific binding to core promoter DNA. Science 264: $933-$ 941.

Vinson, C.R., P.B. Sigler, and S.L. McKnight. 1989. Scissors-grip model for DNA recognition by a family of leucine zipper proteins. Science 246: 911-916.

Wilson, I., H. Niman, R. Houghten, A. Cherenson, M. Connolly, and R. Lerner. 1984. The structure of an antigenic determinant in a protein. Cell 37: 767-778. 


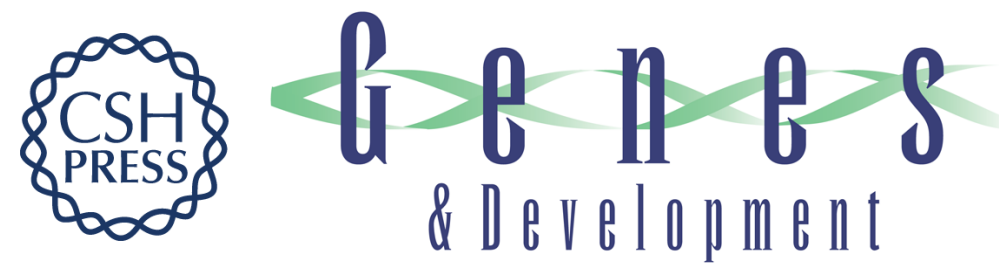

\section{RRN6 and RRN7 encode subunits of a multiprotein complex essential for the initiation of rDNA transcription by RNA polymerase I in Saccharomyces cerevisiae.}

D A Keys, L Vu, J S Steffan, et al.

Genes Dev. 1994, 8:

Access the most recent version at doi:10.1101/gad.8.19.2349

References This article cites 39 articles, 20 of which can be accessed free at:

http://genesdev.cshlp.org/content/8/19/2349.full.html\#ref-list-1

License

Email Alerting

Service

Receive free email alerts when new articles cite this article - sign up in the box at the top right corner of the article or click here.

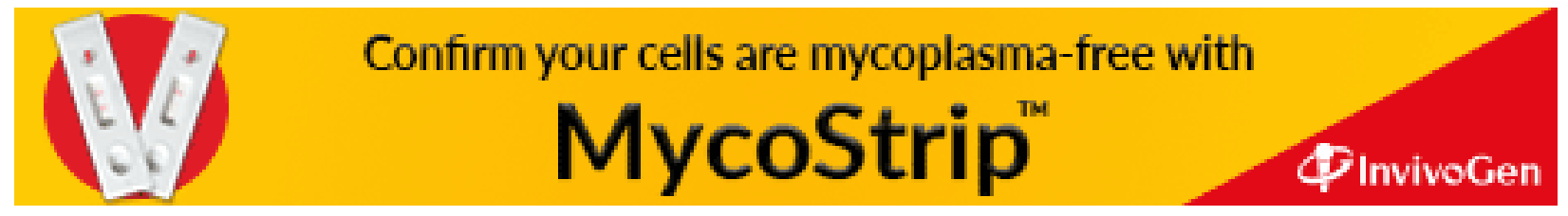

